\title{
Quantum Entanglement Undermines Structural Realism
}

\author{
Forthcoming in Metaphysica \\ Seungbae Park \\ Ulsan National Institute of Science and Technology \\ The Republic of Korea
}

\begin{abstract}
Quantum entanglement poses a challenge to the traditional metaphysical view that an extrinsic property of an object is determined by its intrinsic properties. So structural realists might be tempted to cite quantum entanglement as evidence for structural realism. I argue, however, that quantum entanglement undermines structural realism. If we classify two entangled electrons as a single system, we can say that their spin properties are intrinsic properties of the system, and that we can have knowledge about these intrinsic properties. Specifically, we can know that the parts of the system are entangled and spatially separated from each other. In addition, the concept of supervenience neither illuminates quantum entanglement nor helps structural realism.
\end{abstract}

\section{Keywords}

Intrinsic Property, Quantum Entanglement, Relational Property, Structural Realism, Supervenience

\section{Introduction}

This paper discusses the relationship between quantum entanglement and structural realism. Quantum entanglement is a peculiar phenomenon described by quantum mechanics. Structural realism is a popular view in philosophy of science these days. Does quantum entanglement support or undermine structural realism? It appears initially that quantum entanglement supports structural realism. This paper argues, however, that on close examination, quantum entanglement undermines structural realism.

This present discussion on quantum entanglement and structural realism is organized as follows. In Section 2, I explicate three distinctions crucial to understanding what structural realism asserts: the distinction between an object and a property, the distinction between extrinsic and intrinsic properties, and the distinction between reduction and elimination. In Section 3, I expound structural realism, making use of the three distinctions. In Section 4, I elucidate what it is for two electrons to be entangled, and argue that under the view that the two particles form a single system, the entanglement undermines rather than supports structural realism. I also show that the concept of supervenience neither illuminates quantum entanglement nor helps structural realism. In sum, quantum entanglement cannot be cited as evidence for structural realism.

\section{Three Distinctions}

Traditional metaphysics distinguishes between an object and a property. An object is that which bears a property. The tennis ball bears the property of being spherical and the property of being similar to the baseball. As will become clear in the next section, however, ontic structural realism denies that an object is distinct from a property.

The traditional metaphysics also distinguishes between intrinsic and extrinsic properties. David Lewis says, "We distinguish intrinsic properties, which things have in virtue of the way they themselves are, from extrinsic properties, which they have in virtue of their relations or lack of relations to other things" (Lewis, 1986, p. 61). For example, a tennis 
ball has the intrinsic property of being spherical. The sphericity is a property that the tennis ball has by virtue of the way it is. Also, the tennis ball has the extrinsic property of being similar to a baseball with respect to shape. The similarity is a property the tennis has by virtue of the relation that it bears to the baseball.

Intrinsic properties are ontologically prior to extrinsic properties, and extrinsic properties are derivative of intrinsic properties. For example, the sphericity of the tennis ball and the baseball is ontologically prior to the similarity between the two balls; the similarity derives from their sphericity. Imagine a world in which there is only the tennis ball: In this world, the tennis ball is spherical without being similar to anything else. Also, the similarity of the tennis ball and the baseball is reducible to the sphericity of each of the two balls, i.e., on close examination, the similarity is nothing but the sphericity of each of the two balls. There is no property called "similarity" over and above the sphericity. As we will see in the next section, however, structural realism denies the classical view of the relationship between an intrinsic property and an extrinsic property.

Let me now turn to the distinction between reduction and elimination. We say, for example, that a peach is reducible to a collection of atoms. When we say so, what we mean is that on close examination, the peach turns out to be the collection of atoms. The peach and the collection are not separate objects but rather one and the same object. If God created the collection of atoms, he created the peach, and vice versa. To say that a peach is reducible to the collection of atoms does not mean that the peach does not exist. It only means that what we take to be the peach is nothing but the collection of atoms. So even if the peach is reducible to the collection of atoms, we can still eat a peach.

In contrast, to eliminate an object from our ontology means that we once believed that it existed, but we no longer believe that it exists. For example, witches and phlogiston have been eliminated from our ontology. Paul Churchland (1981) is not a reductionist but rather an eliminativist about beliefs. He does not claim that a belief is reducible to a brain state. He rather claims that beliefs do not exist. The term "phlogiston" is embedded in the false theory, viz., the phlogiston theory, so phlogiston does not exist. Similarly, the term "belief" is embedded in the false theory, viz., folk psychology, so beliefs do not exist. We should eliminate beliefs from our ontology in the way we eliminated witches and phlogiston from our ontology. We will see in the next section that structural realists are eliminative about objects and intrinsic properties.

\section{Structural Realism}

There are diverse versions of structural realism. This paper concerns itself only with epistemic structural realism and ontic structural realism, as defined by James Ladyman (2014). According to epistemic structural realism, "we can believe what scientific theories tell us about the relations entered into by unobservable objects, and suspend judgment as to the nature of the latter" (Ladyman, 2014). In addition, most advocates of epistemic structural realism assert that "there must be individual objects and properties that are ontologically prior to relational structure" (Ladyman, 2014). Thus, epistemic structural realism consists of the metaphysical thesis that there are unobservable objects which have intrinsic and extrinsic properties, and the epistemological thesis that we can know about relational properties of unobservables objects, but not about their intrinsic properties. On this account, objects and their intrinsic properties exist, and they are distinct from their relational properties. Thus, they are neither reducible to relational properties nor eliminable from our ontology. By contrast, ontic structural realism holds "that there are no 'things' and that structure is all there is" (Ladyman, 2014). In other words, objects and intrinsic properties do not exist, and extrinsic properties are all that there is. Objects and intrinsic properties are not reducible to relational 
properties. They should rather be eliminated from our ontology. Thus, they are in the same boat as witches and phlogiston.

Roman Frigg and Ioannis Votsis (2011) observe that the foremost criticism against ontic structural realism is that it is impossible to have relations without relata. Ontic structural realism claims that all that exists are relations. Hence, it is committed to the view that there can be relations without relata. There is a debate over whether it makes sense to say that there can be relations without relata (Ladyman, 2014). Stathis Psillos (2001, 2006), Tian Cao (2003), Jacob Busch (2003), Matteo Morganti (2004) criticize the view, while Howard Stein (1989, p. 59), Simon Saunders (2003), and Ladyman and Don Ross (2007) defend the view. This paper does not explore this territory. It rather explores whether quantum entanglement supports or undermines structural realism.

Before turning to quantum entanglement, however, I want to make a comment on a terminological issue. "Structural realism" is a misnomer. "Relationism" is a better name than "structural realism." The term "structure" usually refers to an internal property of an object. For example, when I say that my body has a certain structure, I mean that my body has parts, and that they are organized in a certain manner. As we noted above, however, structural realism does not assert that an object has certain parts, and that they are organized in a certain manner. It rather asserts that only relations exist, or that we can know only about relations. Why should such a view be called "structural realism" as opposed to "relationism"? "Structural realism" is a misleading name that causes confusion to those who are new to structural realism, but it has been used so widely in the literature for so long that I am stuck with it.

Structural realists might retort that according to structural realism, a structure is a network of relations, networks of relations are all that exists, and that there are no objects and no intrinsic properties. My response to this possible retort is to invite structural realists to consider atomism, according to which a diamond and coal are different objects because they form different networks of atoms. We call this view "atomism," but not "structural realism," because it asserts that atoms are the ultimate reality. By parity of reason, we should call "relationism" the view that relations are the ultimate reality.

Ontic structural realism is an incoherent position. It claims that relations are all that exist. A disconcerting question arises. Can we say that ontic structural realism is true? In epistemology, truth is a relation that a belief bears to the world. If all that exists are relations, as ontic structural realism claims, we can say truths, a kind of relations, exist, but we cannot say that bearers of truths, including ontic structural realism, do not exist. However, it is a contradiction to say that ontic structural realism is true and it does not exist. To put differently, to say that ontic structural realism is true is to admit that there is an object that bears the relational property of being true, viz., ontic structural realism, which implies that ontic structural realism is false. In sum, if it is true, it is false.

Epistemic structural realism is also an incoherent position. It claims that objects exist, but we cannot know about them, and we can only know about relations. It implies that bearers of truths exist, but we cannot know about them, which in turn implies that we can know that something is true, but we cannot know whether that thing is epistemic structural realism. To put differently, to say that we know that epistemic structural realism is true is to admit that there is an object about which we can know, viz., epistemic structural realism. In sum, if it is true, it is false. Therefore, it is just as self-defeating as ontic structural realism.

However, I set aside the self-defeating nature of ontic structural realism and epistemic structural realism. I grant that they are conceptually sound views, and then explore whether quantum entanglement supports or undermines them. 


\section{Quantum Entanglement}

\subsection{Two Quantum Particles}

This section explicates the concept of quantum entanglement in a non-technical manner. An intuitive understanding of this concept is good enough for the purpose of this paper. Let me begin with an everyday example. A light bulb is turned either on or off. It cannot be both on and off at the same time. As this example illustrates, a macroscopic object cannot have two incompatible properties at the same time. Quantum mechanics ${ }^{1}$ tells us, however, that a subatomic particle can probabilistically have two incompatible properties at the same time. For example, an electron is in the superposition of spin-up and spin-down in a certain direction at the same time, i.e., the electron has these two properties indeterminately at the same time. It is $50 \%$ probable that it has the property of spin-up, and also $50 \%$ probable that it has the property of spin-down in a certain direction. When we measure its spin property, however, it definitely comes to have the property of either spin-up or spin-down. That is, it acquires the property of either spin-up or spin-down with $100 \%$ probability. When we do not measure the spin property, however, it has neither the definite property of spin-up nor the definite property of spin-down. It has both the property of spin-up with $50 \%$ probability and the property of spin-down with $50 \%$ probability. We cannot even visualize such a state of affairs.

When quantum mechanics says that an electron has the property of spin-up with $50 \%$ probability, the probability is not a subjective one but rather an objective one. A subjective probability represents the amount of evidence for a belief, whereas an objective probability represents the way the world is. The statement that an electron has the property of spin-up with $50 \%$ probability is intended not to reflect the amount of the evidence for the belief that the electron determinately has the property of spin-up but rather to reflect the state of affairs that the electron probabilistically has the property of spin-up. Again, we cannot even form the image of such a state of affairs.

When two electrons, $e_{1}$ and $e_{2}$, are entangled with each other, the spin property of $e_{1}$ determines the spin property of $\mathrm{e}_{2}$, and vice versa. If we measure the spin property of $\mathrm{e}_{1}$ or $\mathrm{e}_{2}$, both $\mathrm{e}_{1}$ and $\mathrm{e}_{2}$ come to have definite spin properties simultaneously. If we find $\mathrm{e}_{1}$ to have the definite property of spin-up, $\mathrm{e}_{2}$ comes to have the definite property of spin-down. If we find $\mathrm{e}_{2}$ to have the definite property of spin-up, $\mathrm{e}_{1}$ comes to have the definite property of spindown. They come to have the definite spin properties simultaneously. The distance between them does not matter. Even if $e_{1}$ is on the Earth and $e_{2}$ is in the Andromeda Galaxy, the measurement of the spin property of $e_{1}$ instantaneously makes $e_{2}$ have the definite opposite spin property, and vice versa. Albert Einstein called this phenomenon "spooky action at a distance."

\subsection{Relational Properties?}

Quantum entanglement poses a challenge to the traditional metaphysical view that extrinsic properties are determined by intrinsic properties of an object. Alyssa Ney constructs the challenge, without endorsing it, as follows:

When two particles are in the singlet state, neither can individually be said to have a determinate $\mathrm{x}$-spin. So even if spin properties like $\mathrm{x}$-spin up or $\mathrm{x}$-spin down are intrinsic, they are not the sort of properties that particles in states like the singlet state possess. It is rather only the case (given the ontological completeness of the quantum state) for particle 1 that it has the extrinsic property

\footnotetext{
${ }^{1}$ This paper operates under von Neumann and Dirac's version of quantum mechanics. It requires separate papers to explore how structural realism fares under Bohemian mechanics and under the many-worlds interpretation of quantum mechanics.
} 
of having the opposite $\mathrm{x}$-spin of particle 2, and for particle 2 that it has the extrinsic property of having the opposite $\mathrm{x}$-spin of particle 1 . At best, the spin properties attributed to particles by virtue of their entering into entangled state are extrinsic spin properties. (Ney, 2010, p. 227)

The idea is that $\mathrm{e}_{1}$ has the property of spin-up with $50 \%$ probability in virtue of $\mathrm{e}_{2}$ 's having the property of spin-down with $50 \%$ probability, and vice versa. Thus, the spin property of $\mathrm{e}_{1}$ is determined not by an intrinsic property of $e_{1}$ but rather by the spin property of $e_{2}$; the spin property of $\mathrm{e}_{2}$ is determined not by an intrinsic property of $\mathrm{e}_{2}$ but rather by the spin property of $\mathrm{e}_{1}$. In other words, $\mathrm{e}_{1}$ has the spin property not in virtue of the way it is but rather in virtue of the way it relates to $e_{2}$, and $e_{2}$ also has the spin property not in virtue of the way it is but rather in virtue of the way it relates to $e_{1}$. It follows that the spin properties of both $e_{1}$ and $e_{2}$ are not intrinsic properties but rather relational properties.

What are we to make of this argument? It is built upon the metaphysical view that $\mathrm{e}_{1}$ and $\mathrm{e}_{2}$ are two different objects. There is, however, an alternative metaphysical view: $\mathrm{e}_{1}$ and $\mathrm{e}_{2}$ constitute a single object, i.e., $\mathrm{e}_{1}$ and $\mathrm{e}_{2}$ are different parts of the same system. Of course, $\mathrm{e}_{1}$ and $e_{2}$ are spatially separated, so they appear to be separate objects. We can, however, view them as different parts of the same system. Consider, for example, that my heart and liver are spatially separated, but are different parts of the same object, viz., my body. Thus, spatial separation should not pose any problem for the view that $\mathrm{e}_{1}$ and $\mathrm{e}_{2}$ constitute a single system. Under this alternative view, the spin properties of $\mathrm{e}_{1}$ and $\mathrm{e}_{2}$ are intrinsic properties of the system because they are not properties the system has in virtue of the way it relates to another system but rather properties the system has in virtue of the way it is.

Structural realists might argue that the spin properties of $e_{1}$ and $e_{2}$ are not intrinsic properties of the system but rather a structure of the world. In my view, however, this proposal clashes with structural realism, for it implies that the spin properties of $\mathrm{e}_{1}$ and $\mathrm{e}_{2}$ are intrinsic properties of the world. The spin properties cannot be extrinsic properties of the world because the world cannot bear a relation to anything else. If the world can have intrinsic properties, objects in the world can also have intrinsic properties. We have no reason to think that the world can have intrinsic properties, but that its parts cannot. Thus, saying that the spin properties are a structure of the world opens the door to the traditional metaphysical view that objects and intrinsic properties are distinct from extrinsic properties.

Structural realists might object that neither $\mathrm{e}_{1}$ nor $\mathrm{e}_{2}$ has a determinate spin property, so the system as a whole does not have a determinate property, and hence it does not have an intrinsic property. In my view, however, it is a mistake to think that the entanglement of the system is an indeterminate property. It is either $100 \%$ probable or $0 \%$ probable that $\mathrm{e}_{1}$ and $\mathrm{e}_{2}$ are entangled with each other, i.e., they are either definitely in an entangled state or they are not. There is no third state which the system can be in. It is not the case that $\mathrm{e}_{1}$ and $\mathrm{e}_{2}$ are entangled with each other with $50 \%$ objective probability, and that they are not entangled with each other with $50 \%$ objective probability. It is not the system but rather the parts thereof that are in objective probabilistic states. Moreover, the entanglement of $e_{1}$ and $e_{2}$ is not a relational property but rather an intrinsic property of the system, for $\mathrm{e}_{1}$ and $\mathrm{e}_{2}$ are in an entangled state not in virtue of the relation they bear to other electrons but rather in virtue of the way they are.

This discussion shows that once we classify two entangled particles as an object or a system, quantum entanglement undermines rather than supports ontic structural realism. How about epistemic structural realism? Entanglement also undermines it. We can know that two quantum particles are entangled with each other, and that as I argued above, the spin properties of $e_{1}$ and $e_{2}$ are intrinsic properties of the system. Thus, we can have knowledge about the intrinsic properties of the system. 
Let me offer another example of an intrinsic property of a quantum system that we can have knowledge about. Suppose we have two quantum systems: $S_{1}$ and $S_{2}$. $S_{1}$ consists of $e_{1}$ and $e_{2}$ which are entangled with each other. $S_{2}$ consists of $e_{3}$ and $e_{4}$ which are also entangled with each other. Both $e_{1}$ and $e_{3}$ are on the Earth, $e_{2}$ is on Mars, and $e_{4}$ is on Neptune. The two parts of $S_{1}$ are spatially less separated from each other than the two parts of $S_{2}$. Let me call "the entance" the distance between the entangled particles. "Entance" is a portmanteau of "entanglement" and "distance." So $S_{1}$ and $S_{2}$ have different entances, and the entance of $S_{1}$ is shorter than the entance of $S_{2}$. An interesting question arises. Is entance an intrinsic property or a relational property of a quantum system? Of course, it is an intrinsic property. The entance of $S_{1}$ is a property which $S_{1}$ has by virtue of the way it is. It is not a property which $S_{1}$ has by virtue of the relation it bears to $S_{2}$ or any other system. The existence of the entance is a strike against ontic structural realism, according to which a quantum system does not have any irreducible intrinsic property. In addition, we can know about the entance of $S_{1}$ and of $S_{2}$. We can know how far $\mathrm{e}_{1}$ and $\mathrm{e}_{2}$ are separated from each other, and how far $\mathrm{e}_{3}$ and $\mathrm{e}_{4}$ are separated from each other. Our knowledge about the entances is a strike against epistemic structural realism, according to which we cannot know about an irreducible intrinsic property of a quantum system. In sum, the existence of entances and our knowledge about them undermine both ontic structural realism and epistemic structural realism.

My foregoing criticisms against structural realism rely on my classification system according to which two entangled electrons constitute a single system. How would critics respond to my classification system? They might object that I offered no justification for it, and it competes with the traditional classification system according to which two entangled electrons are two objects. Why is my classification system preferable to the traditional classification system?

This objection is reasonable. Let me point out, however, that no justification is offered for the traditional classification system either. In general, there can be no epistemic justification for a classification system, and there can only be a pragmatic justification for it. After all, a classification system is not the kind of thing that can be true or false. It can only be useful or useless. Therefore, my classification system cannot be proved to be false.

\subsection{Supervenience}

A tennis ball is spherical and similar to a baseball with respect to shape. The similarity, an extrinsic property of the tennis ball, is determined by the sphericity, an intrinsic property of the tennis ball. Is the spin property of an electron determined by an intrinsic property of the electron, as the similarity of the tennis ball is determined by the sphericity of the tennis ball? The concept of quantum entanglement implies that the spin property of $\mathrm{e}_{1}$ is determined not by an intrinsic property of $\mathrm{e}_{1}$ but rather by the spin property of $\mathrm{e}_{2}$, and that the spin property of $e_{2}$ is determined not by an intrinsic property of $e_{2}$ but rather by the spin property of $e_{1}$.

Utilizing the peculiarity of quantum entanglement, structural realists might suggest that "in the entangled states of quantum mechanics relations do not supervene on the properties of particles" (Alai, 2017, p. 32). The idea seems to be that the spin property of $\mathrm{e}_{1}$ supervenes not on an intrinsic property of $\mathrm{e}_{1}$ but rather on the spin property of $\mathrm{e}_{2}$; the spin property of $\mathrm{e}_{2}$ supervenes not on an intrinsic property of $\mathrm{e}_{2}$ but rather on the spin property of $\mathrm{e}_{1}$. So quantum entanglement undermines the traditional metaphysical view that an intrinsic property is ontologically prior to an extrinsic property, and it supports the structuralist view that a relational property is not derivative of an intrinsic property. This section attacks both the premise and the form of this argument.

To understand the premise of the argument, we first need to be clear about the concept of supervenience. Simply put, "[w]e have supervenience when there could be no difference of 
one sort without differences of another sort" (Lewis, 1986, p. 14). Suppose that there are two properties: A and B. To say that A supervenes on B means that there can be no difference in A without a difference in B, and that sameness in B implies sameness in A. Thus, B determines A, but not vice versa. For example, to say that pain supervenes on C-fiber firing means that if you feel pain, but I do not, then it must be that you have C-fiber firing, but I do not. It is impossible that both you and I have C-fiber firing. Also, if you and I both have Cfiber firing, then we must both feel pain. It is impossible that you feel pain, but I do not, and vice versa. In short, to say that pain supervenes on $\mathrm{C}$-fiber firing means that $\mathrm{C}$-fiber firing determines pain, but not vice versa. Even if someone feels pain, he might not have C-fiber firing, i.e., it is open whether he has $\mathrm{C}$-fiber firing or not. Imagine that a Martian has a brain made out of silicon as opposed to carbon, so he has not C-fiber firing but rather S-fiber firing. Yet, he can feel pain when his body is damaged. Thus, pain can obtain while C-fiber firing does not, and pain does not determine C-fiber firing. Still, pain supervenes on C-fiber firing.

The concept of supervenience needs to be distinguished from the concept of reduction. As we have seen before, to say that $\mathrm{A}$ is reducible to $\mathrm{B}$ means that on close examination, $\mathrm{A}$ turns out to be $\mathrm{B}$, and that $\mathrm{A}$ and $\mathrm{B}$ are numerically identical with each other, so where there is $\mathrm{A}$, there must be $\mathrm{B}$, and vice versa. For example, Peter Parker is reducible to Spiderman, so where there is Spiderman, so must be Peter Parker, and vice versa. It is impossible that Peter Parker exists, but Spiderman does not, and vice versa. By contrast, to say that A supervenes on B implies that although B determines A, A does not determine B. So where there is B, there must be $\mathrm{A}$, but not vice versa. It follows that $\mathrm{A}$ and $\mathrm{B}$ are distinct from each other. For example, to say that pain supervenes on $\mathrm{C}$-fiber firing implies that where there is pain, there might not be C-fiber firing, so pain and C-fiber firing are distinct from each other. Keep in mind that $\mathrm{A}$ supervenes on $\mathrm{B}$ implies that $\mathrm{A}$ and $\mathrm{B}$ are distinct from each other.

What does it mean to say that the spin property of an electron supervenes on its intrinsic property? It means that if two electrons have qualitatively identical intrinsic properties, and if one electron has the property of spin-up, then the other electron must also have the property of spin-up. In other words, the intrinsic property determines the spin property, i.e., where there is the intrinsic property, there must also be the spin property. It also means that even if two electrons have the same spin property, they might have different intrinsic properties. In other words, the spin property does not determine the intrinsic property, i.e., where there is the spin property, there might not be the intrinsic property. Keep in mind that if the spin property supervenes on the intrinsic property, the spin property and the intrinsic property are different properties.

What does it mean to say that the spin property of an electron does not supervene on its intrinsic property? It means that the spin property does not determine the intrinsic property, and vice versa. Thus, the spin property can exist while the intrinsic property does not, and vice versa. Also, even if two electrons have qualitatively identical intrinsic properties, they may have different spin properties, and even if they have qualitatively identical spin properties, they may have different intrinsic properties. Thus, spin properties and the intrinsic properties are distinct properties, and they have no interesting correlation. This interpretation of non-supervenience goes well with the interpretation that to say that pain does not supervene on $\mathrm{C}$-fiber firing means that pain and C-fiber firing are distinct properties, and that they have no interesting correlation.

As you may have already noticed, it does not matter whether the spin property of an electron supervenes on an intrinsic property of the electron. The spin property and the intrinsic property are different properties. The position that the spin property supervenes on the intrinsic properties of the electron implies that they are different properties. So does the position that the spin property does not supervene on the intrinsic property. The only 
difference between the two positions is that the former position implies that the intrinsic property determines the spin property, whereas the latter position does not.

The implication that the spin property and the intrinsic property are different properties clashes with both ontic and epistemic structural realism. To say that they are different properties presupposes that the intrinsic property exists, and that it is not reducible to an extrinsic property. This presupposition clashes with ontic structural realism that there are no intrinsic properties. In addition, to say that the spin property and the intrinsic property are different properties implies that we know at least one thing about the intrinsic property, viz., it is different from the spin property. This corollary clashes with epistemic structural realism that we cannot know anything about the intrinsic properties of unobservable objects.

Can structural realists contend that the spin property of $\mathrm{e}_{1}$ supervenes on the spin property of $\mathrm{e}_{2}$ ? The answer at first appears to be "Yes," given that the spin property of $\mathrm{e}_{2}$ determines the spin property of $\mathrm{e}_{1}$. On close examination, however, the answer is "No" because to say "Yes" implies that the spin property of $\mathrm{e}_{1}$ does not determine the spin property of $\mathrm{e}_{2}$. Recall that to say that A supervenes on B implies that A does not determine B. This implication runs counter to the idea that if $e_{1}$ is entangled with $e_{2}$, the spin property of $e_{1}$ determines the spin property of $\mathrm{e}_{2}$. Consequently, it is wrong to say that the spin property of $\mathrm{e}_{1}$ supervenes on the spin property of $\mathrm{e}_{2}$.

Can structural realists say that the spin property of $\mathrm{e}_{1}$ supervenes on the spin property of $\mathrm{e}_{2}$, and that the spin property of $\mathrm{e}_{2}$ supervenes on the spin property of $\mathrm{e}_{1}$ ? Two contradictions are involved in saying "Yes." To say that the spin property of $\mathrm{e}_{1}$ supervenes on the spin property of $e_{2}$ implies that the spin property of $e_{2}$ determines the spin property of $e_{1}$, but that the spin property of $\mathrm{e}_{1}$ does not determine the spin property of $\mathrm{e}_{1}$. To say that the spin property of $\mathrm{e}_{2}$ supervenes on the spin property of $\mathrm{e}_{1}$ implies that the spin property of $\mathrm{e}_{1}$ determines the spin property of $\mathrm{e}_{2}$, but that the spin property of $\mathrm{e}_{2}$ does not determine the spin property of $\mathrm{e}_{1}$. It is a contradiction to say that the spin property of $\mathrm{e}_{2}$ determines the spin property of $e_{1}$, and that the spin property of $e_{2}$ does not determine the spin property of $e_{1}$. It is also a contradiction to say that the spin property of $\mathrm{e}_{1}$ does not determine the spin property of $\mathrm{e}_{1}$ and the spin property of $\mathrm{e}_{1}$ determines the spin property of $\mathrm{e}_{2}$.

So far in this section, I have criticized the premise of the possible structural realists' argument. Let me now turn to the relation between the premise and the conclusion. It is a fallacious argument that since a spin property of an electron does not supervene on the intrinsic properties of electrons, an intrinsic property does not exist. Imagine that a philosopher of mind contends that since pain does not supervene on C-fiber firing, C-fiber firing does not exist. Such an argument would fly in the face of philosophers of mind. Unfortunately, there is no structural difference between the structural realists' argument and the counterintuitive argument made.

\section{Conclusion}

The spin property of $\mathrm{e}_{1}$ is determined not by its intrinsic property but rather by the spin property of $e_{2}$. So structural realists might cite quantum entanglement as evidence for structural realism. I argued, however, that once we view two entangled electrons as constituting a single system, the entanglement of the two electrons undermines rather than supports structural realism. Ontic structural realism is undermined by the suggestion that the spin properties are intrinsic properties of the system. Epistemic structural realism is undermined by the suggestion that we know that two electrons are entangled with each other, and that they are spatially separated from each other.

Structural realists might contend that the spin property of a quantum particle does not supervene on an intrinsic property of the quantum particle. I argued that their contention 
destroys structural realism, for it presupposes both that extrinsic and intrinsic properties are distinct properties, and that we can have knowledge about intrinsic properties. Moreover, it is an objectionable inference that since the spin property of an electron does not supervene on an intrinsic property of the electron, the intrinsic property does not exist. The concept of supervenience neither illuminates quantum entanglement nor helps structural realism.

To sum up, although quantum entanglement poses a challenge to the traditional metaphysical view that an extrinsic property is determined by an intrinsic property of an object, it supports neither ontic structural realism that all that exists are relational properties nor epistemic structural realism that we cannot know about the intrinsic property of quantum particles. As I said earlier, however, there are other versions of structural realism. Also, there are other arguments for and against structural realism. They should be addressed in separate papers. This paper only concerns the issue of whether quantum entanglement supports ontic structural realism and epistemic structural realism.

\section{References}

Alai, M. (2017). The debates on scientific realism today: Knowledge and objectivity in science. In E. Agazzi (Ed.), Varieties of scientific realism: Objectivity and truth in science. (pp. 19-47). Cham: Springer.

Busch, J. (2003). What structures could not be. International Studies in the Philosophy of Science, 17(3), 211-225.

Cao, T. (2003). Between platonism and phenomenalism: Reply to Cao. Synthese, 136(1), 7378.

Churchland, P. (1981). Eliminative materialism and the propositional attitudes. Journal of Philosophy, 78(2), 67-90.

Frigg, R. and I. Votsis (2011). Everything you always wanted to know about structural realism but were afraid to ask. European Journal for Philosophy of Science, 1(2), 227-276.

Ladyman, J. (2014). Structural realism. The Stanford Encyclopedia of Philosophy, E. Zalta (ed.), URL $=\langle$ http://plato.stanford.edu/archives/spr2014/entries/structural-realism/>.

Ladyman, J. and D. Ross, D. Spurrett, and J. Collier (2007). Every thing must go: Metaphysics naturalised. Oxford: Oxford University Press.

Lewis, D. (1986). On the plurality of worlds. Malden, Massachusetts: Blackwell Publishing.

Morganti, M. (2004). On the preferability of epistemic structural realism. Synthese 142(1), 81-107.

Ney, A. (2010). Are there fundamental intrinsic properties? In A. Hazelett (Ed.), New waves in physics. (pp. 219-239). New York: Palgrave Macmillan:.

Psillos, S. (2001). Is structural realism possible? Philosophy of Science, 68(Supplementary Volume), S13-S24. 
Psillos, S. (2006). The structure, the whole structure and nothing but the structure? Philosophy of Science 73(5), 560-570.

Saunders, S. (2003). Structural realism again. Synthese, 136(1), 127-133.

Stein, H. (1989). Yes, but... some skeptical remarks on realism and antirealism. Dialectica 43(1), 47-65. 